\title{
Characterizing Fruit Fly Flight Behavior Using a Microforce Sensor With a New Comb-Drive Configuration
}

\author{
Yu Sun, Member, IEEE, Member, ASME, Steven N. Fry, D. P. Potasek, Dominik J. Bell, and \\ Brad J. Nelson, Member, IEEE, Member, ASME
}

\begin{abstract}
This paper reports a MEMS microforce sensor with a novel configuration of bulk micromachined differential triplate comb drives that overcomes the difficulty of electrically isolating the two stationary capacitor comb sets in bulk micromachining. A high-yield fabrication process using deep-reactive ion etching (DRIE) on silicon-on-insulator (SOI) wafers and only three lithographic masks was utilized to construct the high aspect ratio devices. The process features dry release of both suspended structures and the entire device in order to protect fragile components. The sensor has a high sensitivity $(1.35 \mathrm{mV} / \mu \mathrm{N})$, good linearity $(<4 \%)$, and a large bandwidth $(7.8 \mathrm{kHz})$, and is therefore well suited for characterizing flight behavior of fruit flies (Drosophila melanogaster). The technique allows for the instantaneous measurement of flight forces, which result from a combination of aerodynamic forces and inertial forces generated by the wings, and demonstrates a novel experimental paradigm for exploring flight biomechanics in the fruit fly. The average lift force is determined to be $9.3 \mu \mathrm{N}( \pm 2.3 \mu \mathrm{N})$, which is in the range of typical body weights of fruit flies. The potential impact of this research extends beyond gathering flight data on Drosophila melanogaster by demonstrating how MEMS technology can be used to provide valuable tools for biomechanical investigations.

[1208]
\end{abstract}

Index Terms-Capacitive sensor, deep-reactive ion etching (DRIE) on silicon-on-insulator (SOI), dice free dry release, differential triplate comb drive, Drosophila melanogaster, fruit flies, high aspect ratio, microforce sensing.

\section{INTRODUCTION}

A $\mathrm{S}$ SCIENTISTS and engineers strive to develop more intelligent microrobotic systems, many in the field are increasingly turning toward biological organizms in order to obtain design inspiration. For example, researchers have considered cockroaches [1], [2], crickets [3], and earthworms [4] to guide the design of small autonomous microsystems. Over the past few years, the flight behavior of small insects has attracted interest for the development of flying microrobotic

Manuscript received November 25, 2003; revised April 30, 2004. This work was supported in part by the U.S. National Science Foundation under Grant IIS-0208564. Subject Editor A. J. Ricco.

Y. Sun is with the Department of Mechanical and Industrial Engineering and the Institute of Biomaterials and Biomedical Engineering, University of Toronto, Toronto, ON M5S 3G8, Canada (e-mail: sun@mie.utoronto.ca).

S. N. Fry is with the Institute of Neuroinformatics, Swiss Federal Institute of Technology (ETH) and University of Zürich, Switzerland

D. P. Potasek is with the Department of Mechanical Engineering, University of Minnesota, Minneapolis, MN 55455 USA.

D. J. Bell and B. J. Nelson are with the Institute of Robotics and Intelligent Systems, Swiss Federal Institute of Technology (ETH), Zürich, Switzerland

Digital Object Identifier 10.1109/JMEMS.2004.839028

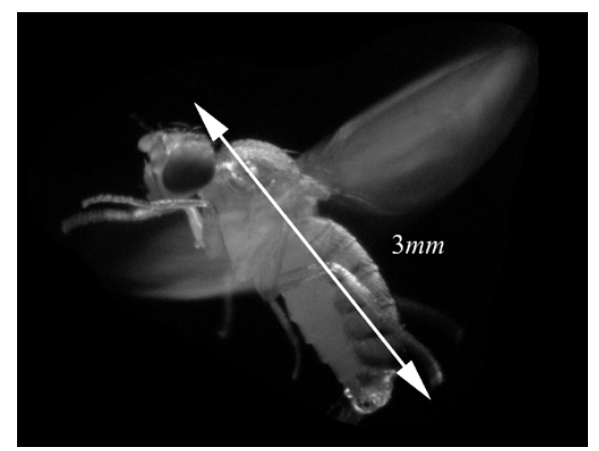

Fig. 1. Drosophila melanogaster in typical hovering posture.

systems [5], [6]. One such insect that is particularly interesting from the standpoint of complete flight system design is the fruit fly Drosophila melanogaster.

The fruit fly, shown in Fig. 1, is a model organism studied by biologists for almost a century, and possesses a highly developed flight control system that provides the insect with the capability to perform robust stable flight, as well as exceedingly rapid and precise turning maneuvers. The neurophysiology and biomechanics are inextricably linked and must be considered at the systems level. Multimodal sensory input converges on only 18 control muscles [7] that are responsible for the finetuning of wing motion for maneuvering, the aerodynamic basis of which has recently been revealed [8]. Beyond its impressive flight behavior, the fact that Drosophila melanogaster is completely autonomous, extremely small, highly robust, and self replicating makes this organizm particularly interesting from a microrobotics standpoint [9].

To better understand the biomechanics underlying fly flight, precise measurements of the flight forces of these tiny ( $3 \mathrm{~mm}$ long) insects must be obtained. Three-dimensional (3-D) high-speed videography allows a precise measurement of wing and body kinematics in free flight [8], which can then be played through a dynamically scaled robotic wing model [10] to obtain the aerodynamic forces. Furthermore, the inertial forces resulting from wing acceleration can be estimated from the measured wing position. However, the estimation of inertial forces is error prone and the analysis of large amounts of image data is prohibitively cumbersome and time-consuming. Therefore, a robust real-time method for direct flight force measurements is desired. Such measurements have previously been made by measuring flight forces exerted by flies tethered 

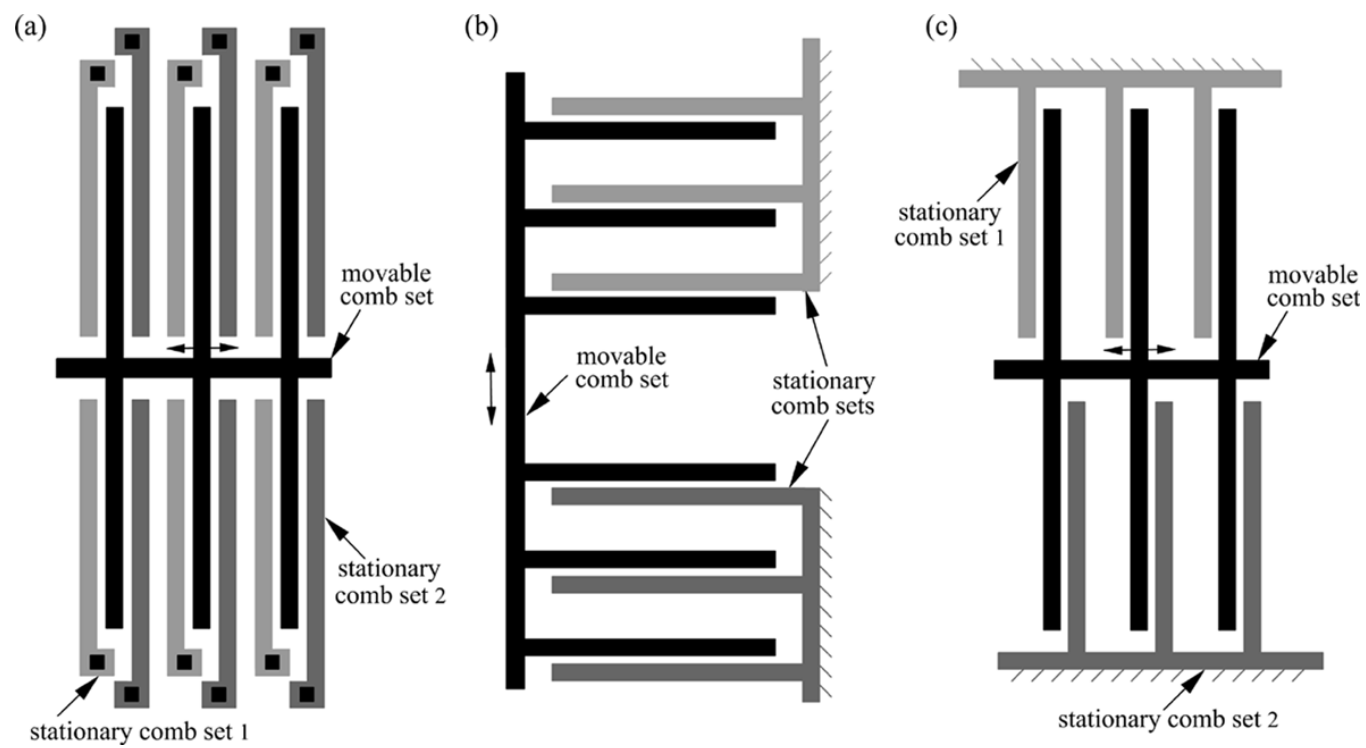

Fig. 2. (a) Surface micromachined differential comb drives. (b) Bulk micromachined differential comb drives consisting of two connected capacitor pairs [15]. (c) Differential triplate comb drive configuration used in this design that is suitable for bulk micromachining.

on a steel wire. Displacements caused by the forces were measured using laser interferometry [11]. In principle, this method is suitable for measuring the instantaneous flight forces in the fly's sagittal plane; however, measurement artifacts due to resonance issues were also reported. Although it allows detailed measurements, this method has not found broad application in biomechanical studies, possibly due to the relative complexity of the setup.

MEMS technology provides the opportunity to develop much smaller and inexpensive microforce sensors with a high sensitivity and large bandwidth. Importantly, sensor mechanical properties are well-defined, allowing novel sensor designs to be implemented. Due to their small size, MEMS-based microforce sensors can be readily integrated into existing experimental setups and, therefore, provide a significantly enhanced data acquisition technology for biomechanical research. In this paper, a MEMS microforce sensor is presented that is suitable for measuring the instantaneous lift forces generated by fruit flies. The potential impact of this research extends beyond the presented application by promising valuable tools for a broad range of biomechanical applications.

\section{MEMS MicROFORCE SENSING}

Various MEMS force sensor designs have been proposed and realized. Microforce sensing techniques can be classified into different categories based on the relationships between mechanical forces (or mechanical deformation) and sensory properties, including piezoresistive, piezoelectric, capactive, optical, and magnetic. Among these sensing mechanisms, capacitive microforce sensing has the advantage of low power, low noise, high sensitivity, and insensitivity to temperature variation. The common configurations are lateral comb drives (overlapping area changes) [12] and transverse comb drives (gap changes) [13]. Compared to the lateral configuration, transverse comb drives have higher sensitivity but suffer from significant nonlinearity. The comb drives described in this paper

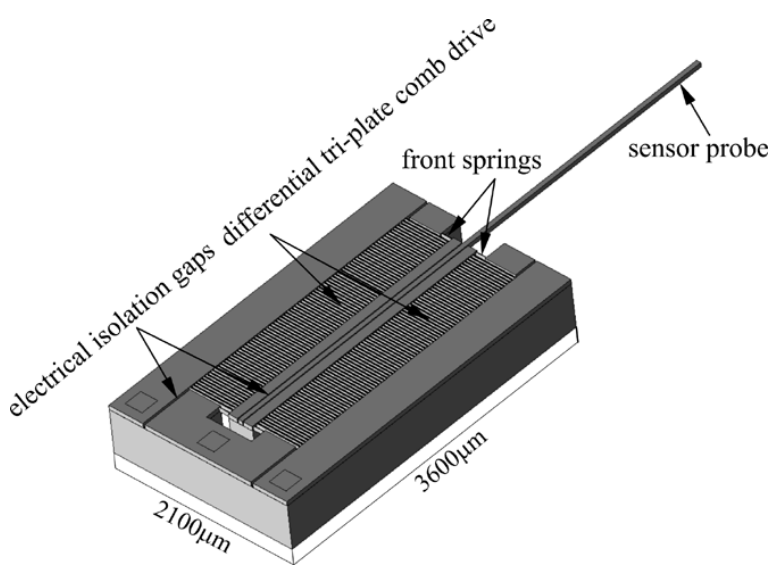

Fig. 3. Capacitive microforce sensor solid model.

are of the transverse orientation; however, they provide both the high sensitivity and linearity that traditional transverse comb drives lack. Differential tri-plate comb drives have been used, for example, in the ADXL50 accelerometer [14]. ADXL50's surface micromachined comb-drive configuration, as shown in Fig. 2(a), is easily realized in surface micromachining; however, it is not suitable for production by bulk micromachining due to the difficulty of electrically isolating the two stationary capacitor comb sets in bulk micromachining. Because bulk micromachining is capable of constructing higher aspect ratio comb drives for improving sensitivity, two connected capacitor pairs shown in Fig. 2(b) were used to construct differential comb drives that were bulk micromachined [15]. In this paper, the configuration shown in Fig. 2(c) is used to form the differential tri-plate comb drive that is suitable for production using bulk micromachining, which is achieved by separating the two stationary capacitor comb sets on either side of the movable comb set.

Many processes have been developed to form high aspect ratio comb drive structures including the SCREAM process [16], the dissolved wafer process [17], the etch-diffusion 
(a)

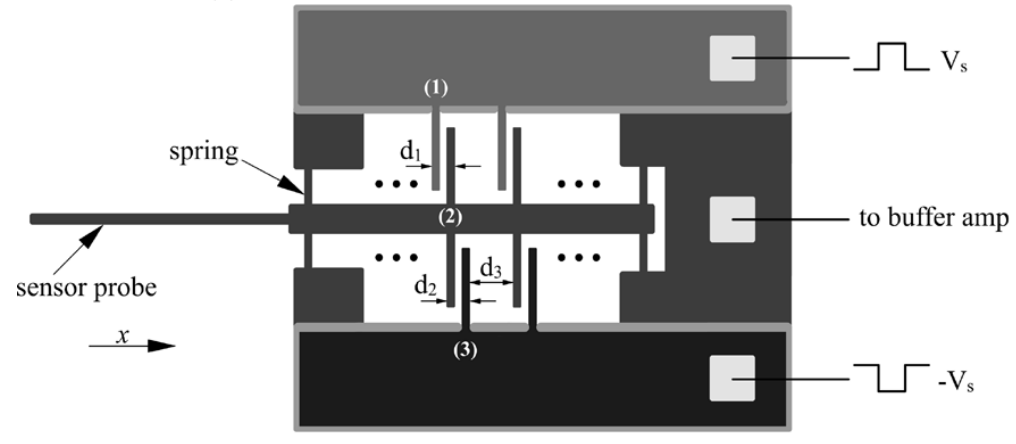

(b)

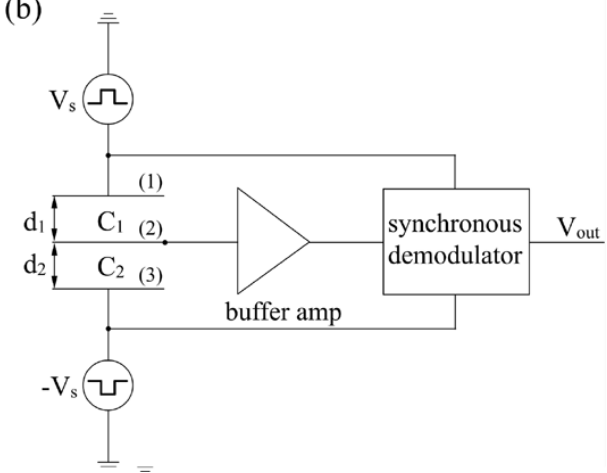

Fig. 4. (a) Microforce sensor schematic with differential triplate comb drives. (b) Block diagram of sensor and readout circuitry.

process [18], the surface/bulk micromachining process [19], and molding processes such as LIGA [20] and HEXIL [21]. The high aspect ratio device described in this paper was constructed by a simple and high-yield fabrication process using DRIE on SOI wafers [13]. A similar process, the DRIE and sealed cavity wafer bonding process, was earlier reported [15], [22], [23] in which cavities are etched on a handle layer Si substrate before fusion bonding so that the structures on the device layer can be suspended after release. In order to reduce the pressure inside the cavities, fusion bonding was required to be done either in an oxygen-rich ambient [22] or in a vacuum if the device layer $\mathrm{Si}$ is thinner than $20 \mu \mathrm{m}$. In this process [13], the handle layer $\mathrm{Si}$ substrate does not need to be patterned before fusion bonding. The handle layer is an integrative part of the force sensor, used for structural stability, dice free releasing of the fragile structures, suspending the comb capacitors, as well as mechanically connecting and electrically isolating capacitor plates, which is shown in Fig. 3. The process also features dry release of both suspended structures (as in [24]) and the entire device in order to protect fragile components.

\section{MicROfORCE SENSOR DESIGN}

Fig. 3 shows a solid model of the micro force sensor design. The sensor probe transmits forces axially deflecting the unidirectionally compliant springs. This deflection displaces the inner movable capacitor plates - plates (2) shown in Fig. 4(a). With a force applied in the positive $\mathrm{x}$ direction, plates (2) move away from plates (1) and closer to plates (3). When an ac signal is applied to the outer capacitors [plates (1) and (3)], a voltage divider is formed, as shown in Fig. 4(b).

The resulting signal $V_{\text {out }}$ is

$$
V_{\mathrm{out}}=V_{s}\left(\frac{C_{1}-C_{2}}{C_{1}+C_{2}}\right)
$$

where $C_{1}=K \varepsilon\left(A_{1} / d_{1}\right)+K \varepsilon\left(A_{1} / d_{3}\right), C_{2}=K \varepsilon\left(A_{2} / d_{2}\right)+$ $K \varepsilon\left(A_{2} / d_{3}\right), K$ is dielectric constant for the ambient (for air $K=1), \varepsilon=8.8542 \times 10^{-12} \mathrm{C}^{2} /\left(\mathrm{N} \times \mathrm{m}^{2}\right)$ is the permittivity of free space, and overlapping plate area $A_{1}=A_{2}$. The plates are nominally spaced equally at $d_{0}=\left(d_{1}+d_{2}\right) / 2$. The plate distances are, thus, defined as $d_{1}=d_{0}+\Delta d$ and $d_{2}=d_{0}-\Delta d$, where $\Delta d$ is the displacement of the middle plate. By initially setting $d_{1}=d_{2} \ll d_{3}$, the undesired additional parallel capacitance effect is minimized, and linearity is maintained. From (1), the resulting output signal $V_{\text {out }}$ can be shown to be proportional to the middle plate displacement.

$$
V_{\mathrm{out}}=V_{s}\left(\frac{\Delta d}{d_{0}}\right)
$$

Fig. 5 shows the simulated deflection-output relationship for $d_{3}=6,8,20 \mu \mathrm{m}$ (in all cases $d_{1}=d_{2}=5 \mu \mathrm{m}$ ). It can be seen that repeating the comb drive unit reasonably far apart makes the undesired parallel capacitance effect negligible and maintains system linearity.

The stiffness of the sensors is determined by the spring dimensions. The springs are modeled as two fixed-fixed beams with a point load applied in the middle. The force-deflection model is

$$
\Delta d=\frac{F l^{3}}{4 E w^{3} t}
$$

where $F$ is the total applied force, $E=100 \mathrm{GPa}$ is the average Young's modulus of P-type $\langle 100\rangle$ silicon, and $l, w$, and $t$ are spring length, width, and thickness.

\section{MICROFABRICATION}

Fig. 6 illustrates the microfabrication process. Step A to Step D can be replaced by directly purchasing commercial SOI wafers. After Step E, the wafer is fragile due to the deep trenches on the backside; however, the $50 \mu \mathrm{m}$ top Si layer is study enough for subsequent processing from the support of the remaining $\mathrm{Si}$ on the handle layer.

In this design and process, the handle layer $\mathrm{Si}$ is an integrative part of the force sensor, used for structural stability, dice free releasing of the fragile structures, suspending the comb capacitors, as well as mechanically connecting and electrically isolating capacitor plates, which is shown in Fig. 3. The process also features dry release of both suspended structures and the entire device in order to protect fragile components. The comb capacitor plates are $50 \mu \mathrm{m}$ in depth, greatly increasing device sensitivity because of the large overlapping area. An aspect ratio of more than 100 can be achieved using the microfabrication 


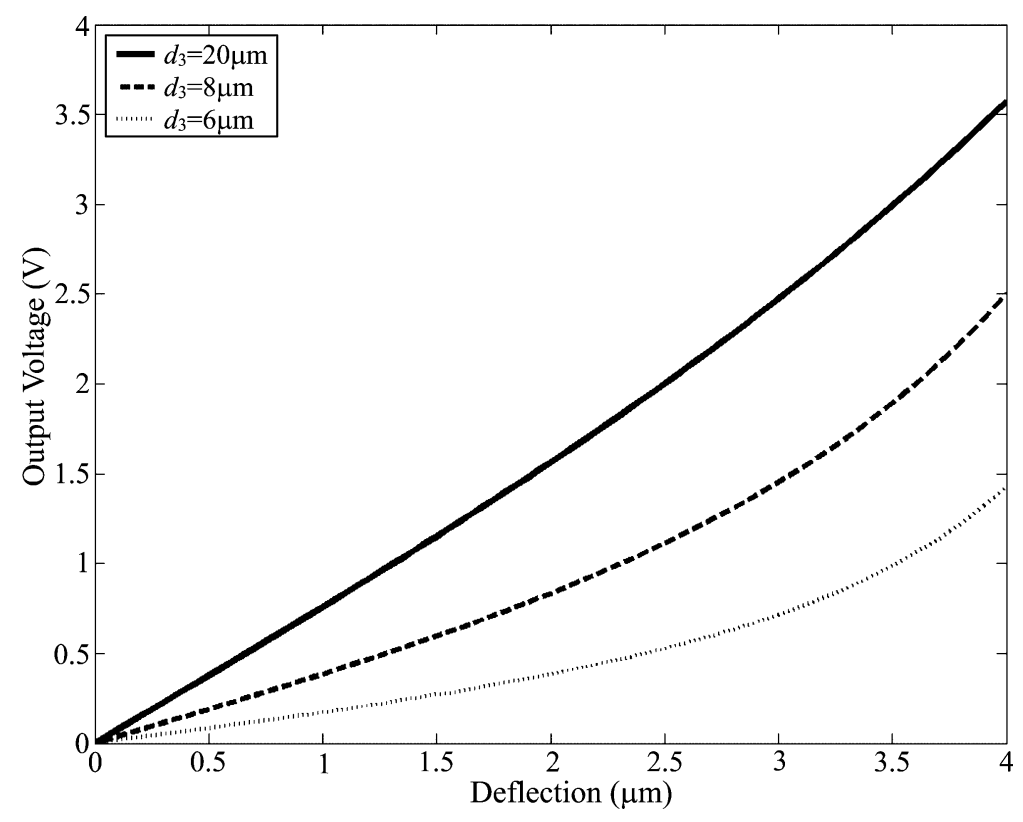

Fig. 5. Simulation results of linearity comparison by varying capacitor gap $d_{3}$, assuming $l=125 \mu \mathrm{m}, w=5 \mu \mathrm{m}$, and $t=50 \mu \mathrm{m}$.

A
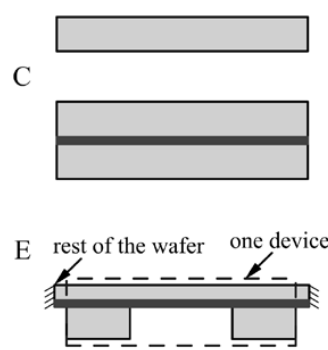

G

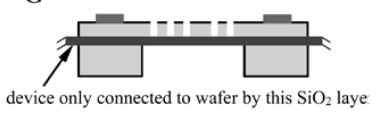

B

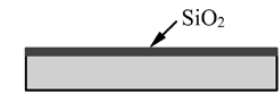

D

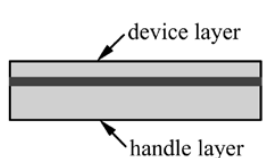

F

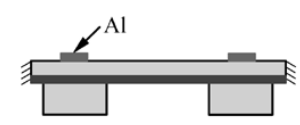

$\mathrm{H}$

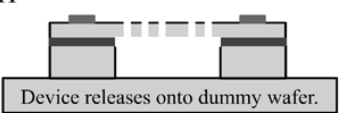

Fig. 6. Microfabrication process.Step A. Start from a double polished P-type wafer with crystal orientation of $\langle 100\rangle$. Step B. LPCVD (Low pressure chemical vapor deposition) $1 \mu \mathrm{m} \mathrm{SiO}_{2}$. Step C. Fusion bond the wafer with $\mathrm{SiO}_{2}$ with another P-type wafer. Step D. CMP (Chemical mechanical polishing) the top wafer (the device layer) down to $50 \mu \mathrm{m}$. This forms an SOI (silicon-on-insulator) wafer. Step E. DRIE (Deep reactive ion etching) to form the features on the back side (the handle layer) such as the outer frame and inner movable structure. The buried $1 \mu \mathrm{m} \mathrm{SiO} \mathrm{S}_{2}$ layer acts as an etch stop layer and also as an insulator between the capacitors. A PlasmaTherm SLR-770 Inductively Coupled Plasma Reactive Ion Etcher was used in processing. Step F. E-beam evaporate Al to form ohmic contacts; liftoff to pattern Al. Step G. DRIE the top side to form capacitive comb fingers and springs. The devices were connected to the device wafer only by the buried $\mathrm{SiO}_{2}$ layer. Step $\mathrm{H}$. RIE (Reactive ion etching) to remove the buried $\mathrm{SiO}_{2}$ layer. The devices released onto a carrier dummy wafer below the device wafer, and then were picked up individually from the carrier dummy wafer. The dice-free release process protects fragile structures from damage.

process, which requires only three lithographic masks. Fig. 7 shows an SEM picture of a completed device.

\section{CALIBRATiON}

The excitation waveform of the readout circuit is a $1 \mathrm{MHz}$ square wave nominally at $5 \mathrm{~V}$. The circuit utilizes a buffer amplifier leading into a synchronous demodulator which supplies feedback to the drive voltage. Calibration was conducted using a microscale (AG285 DeltaRange). Fig. 8 shows the calibration results, and device specifications are summarized in Table I.

The electrostatic force generated from the excitation voltages is shown in (4) at the bottom of the page where $N$ is the number of repeating comb units, $A$ is the plate area, and $V$ is the applied voltage.

The electrostatic force generated is $0.019 \mu \mathrm{N}$ when a $10 \mu \mathrm{N}$ force is applied. This undesired force is negligible considering the device resolution and measurement range, and can be further reduced by applying excitation signals of lower magnitude.

\section{Drosophila Flight Force MEASUREMENT}

The force sensor has been applied to characterizing the flight forces produced by tethered fruit flies. For the experiments, a stock of Drosophila melanogaster derived from a wild-caught base population was used. Fruit flies are particularly well adapted for flight and provide an ideal model for studying the neurophysiology underlying flight control.

In the experiments, individual flies were attached to the MEMS sensor probe following the procedure described in [25]. First, the fly was immobilized on a custom machined stage cooled by a thermostat controlled Peltier element to $4{ }^{\circ} \mathrm{C}$. Second, a drop of UV sensitive glue (Loctite, Duro Clear Glass Adhesive) with an approximate diameter of $50 \mu \mathrm{m}$ was applied

$$
F_{e}=2 N A \varepsilon V^{2} \Delta d\left[\frac{d_{0}}{\left(d_{0}-\Delta d\right)^{2}\left(d_{0}+\Delta d\right)^{2}}+\frac{d_{3}}{\left(d_{3}-\Delta d\right)^{2}\left(d_{3}+\Delta d\right)^{2}}\right]
$$


(a)

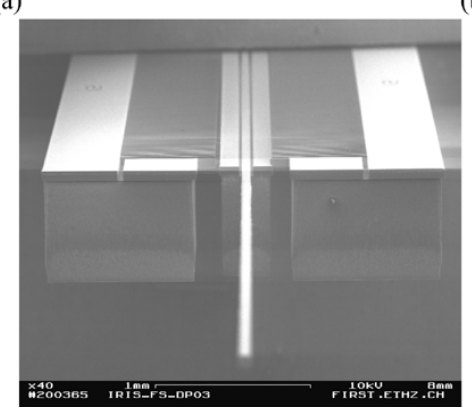

(b)

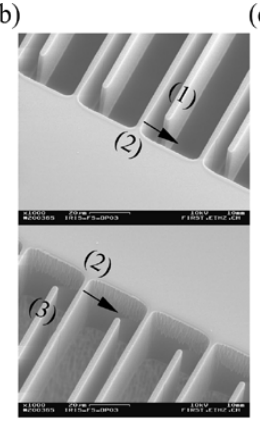

(c)

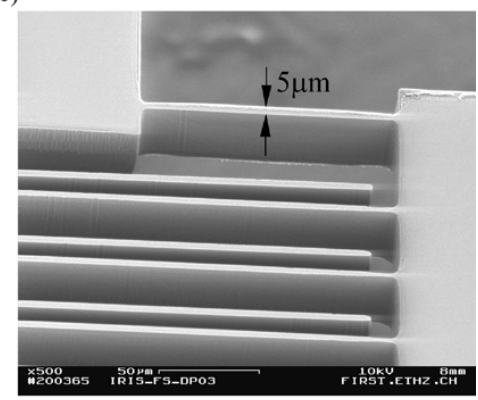

Fig. 7. (a) SEM of a force sensor. (b) Differential triplate comb drives. (c) Suspended spring and comb drives.

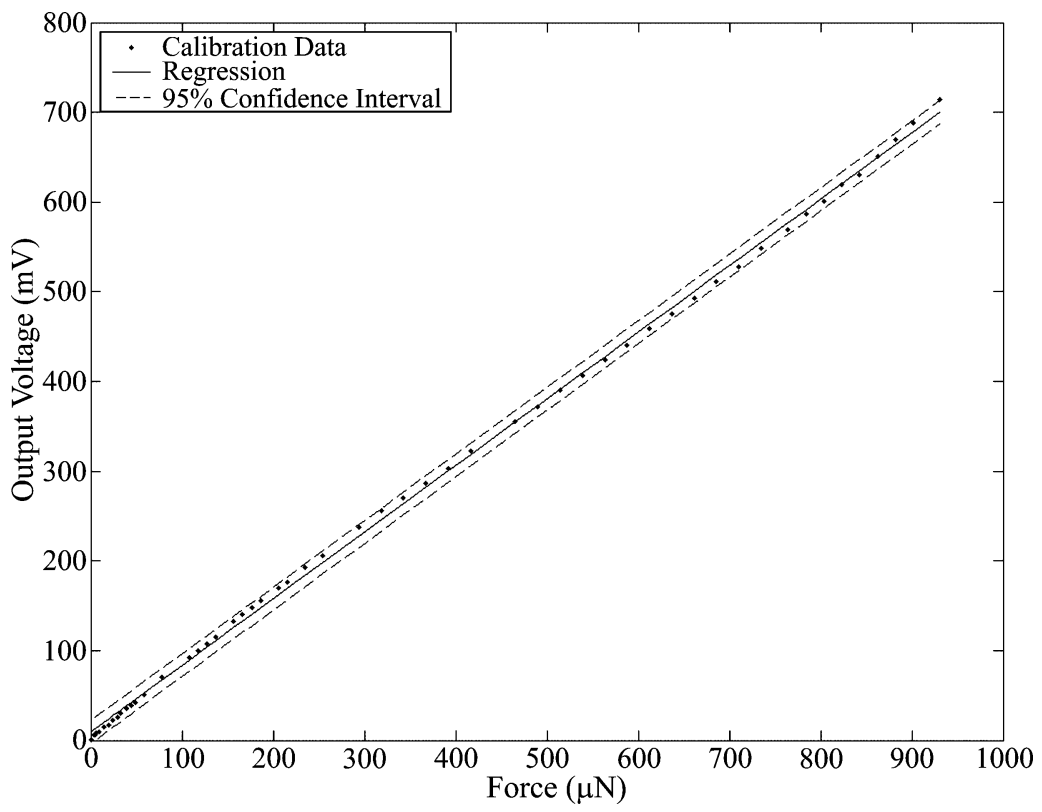

Fig. 8. Microforce sensor calibration results under static loads.

TABLE I

DEVICE SPECIFICATIONS

\begin{tabular}{|c|c|c|}
\hline \multicolumn{2}{|c|}{ resolution } & $0.68 \mu \mathrm{N}$ \\
\hline & sensitivity & $1.35 \mathrm{mV} / \mu \mathrm{N}$ \\
\hline & stiffness & $1280 \mathrm{~N} / \mathrm{m}$ \\
\hline \multicolumn{2}{|c|}{ measurement range } & $+/-1 \mathrm{mN}$ \\
\hline \multicolumn{2}{|r|}{ bandwidth } & $7.8 \mathrm{kHz}$ \\
\hline \multicolumn{2}{|r|}{ linearity } & $<4 \%$ \\
\hline \multirow{6}{*}{ 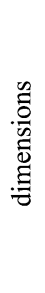 } & base & $3.6 \mathrm{~mm} \times 2.1 \mathrm{~mm} \times 0.5 \mathrm{~mm}$ \\
\hline & probe & $3 \mathrm{~mm} \times 50 \mu \mathrm{m} \times 50 \mu \mathrm{m}$ \\
\hline & cap. gap $\left(d_{1}, d_{2}\right)$ & $5 \mu \mathrm{m}$ \\
\hline & cap. spacing $\left(d_{3}\right)$ & $20 \mu \mathrm{m}$ \\
\hline & cap. plates & $500 \mu \mathrm{m} \times 5 \mu \mathrm{m} \times 50 \mu \mathrm{m}$ \\
\hline & springs & $125 \mu \mathrm{m} \times 5 \mu \mathrm{m} \times 50 \mu \mathrm{m}$ \\
\hline
\end{tabular}

to the fly's thorax using a thin tungsten probe mounted on a micromanipulator (Sutter MP285). Third, the MEMS sensor probe was brought into contact with the glue, which was fully cured with a UV light gun (ELC305). Finally, the sensor with the attached fly was lifted away from the mounting stage. After the fly's body warmed up, typically within a few minutes, the

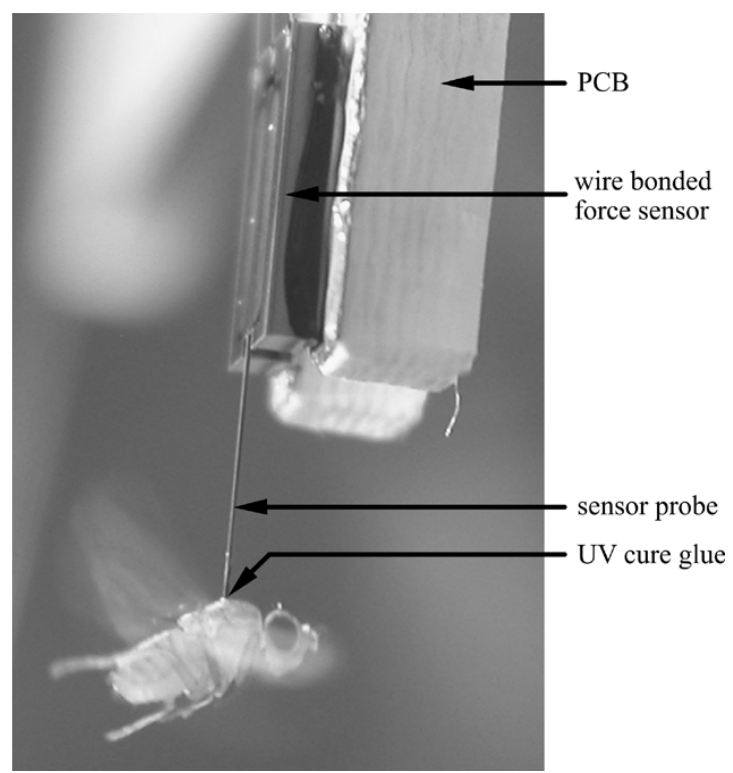

Fig. 9. Flight force sensing of Drosophila melanogaster tethered to sensor probe.

fly initiated tethered flight either spontaneously or after a puff of air was applied. Fig. 9 shows a fruit fly tethered to the force sensor probe and the force sensor wire bonded to a PCB. 


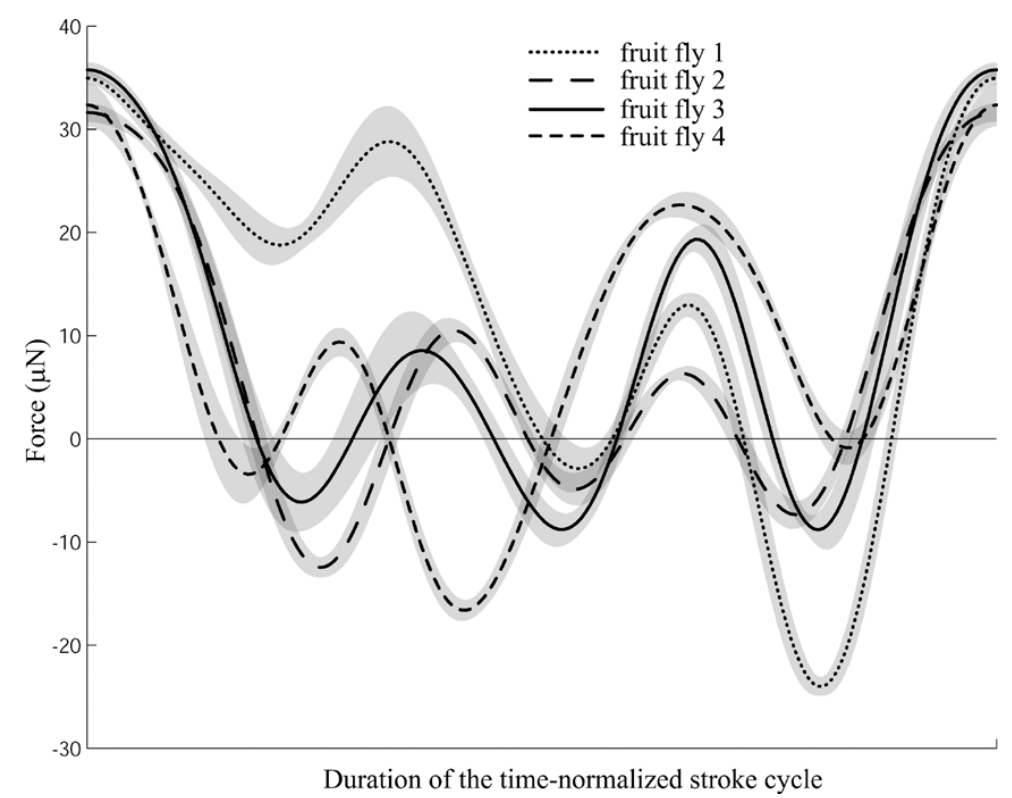

Fig. 10. Flight lift forces from four flies over the duration of the time-normalized stroke cycle. The gray areas show $95 \%$ confidence intervals.

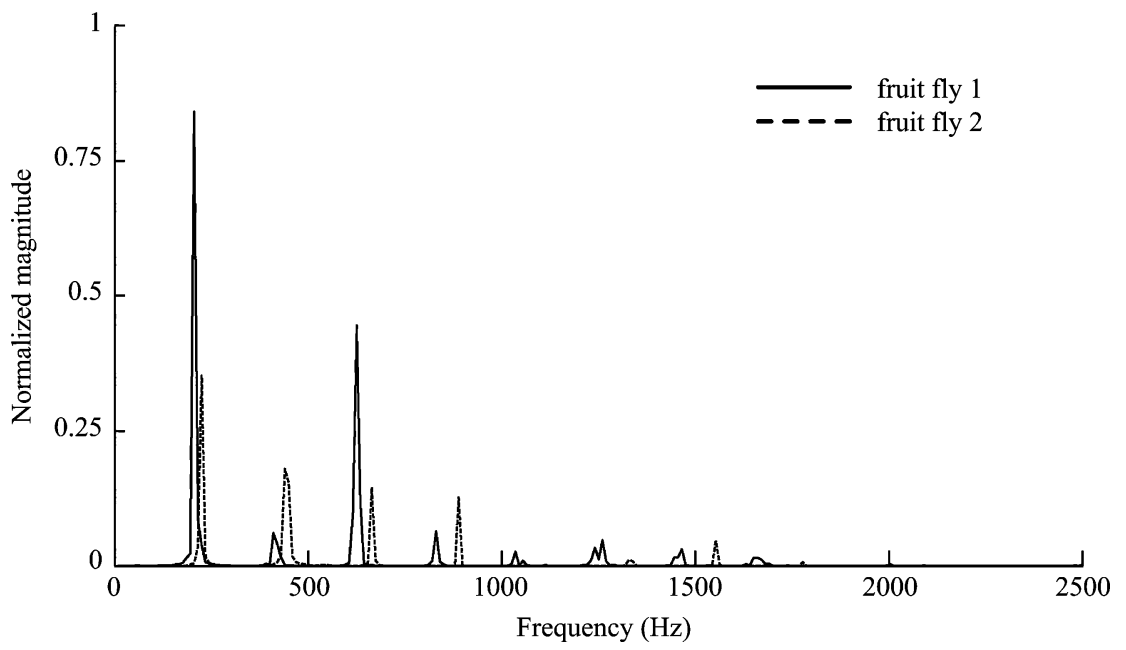

Fig. 11. FFT analysis results of data sets from two fruit flies.

Data were collected from six fruit flies at a sampling rate of $5 \mathrm{kHz}$. The measured signal is periodic, with a fundamental frequency just above $200 \mathrm{~Hz}$, corresponding to the typical wing beat frequency of fruit flies. The data were filtered using a fourth-order zero phase-lag digital Butterworth filter with a cutoff frequency of $1 \mathrm{kHz}$ and upsampled using a B-spline algorithm. Two samples that did not reveal consistent flight behavior throughout the recorded period were discarded. Fig. 10 shows the average lift forces from four flies over the duration of the time-normalized stroke cycle based on a total of 2875 wing strokes. Within each sequence, a contiguous section that showed little variance in wing beat frequency was chosen. The average lift force is $9.3 \mu \mathrm{N}( \pm 2.3 \mu \mathrm{N})$, which is in the range of typical body weights of fruit flies.

The phase of the forces between samples and with respect to the wing stroke is not clearly known. The flight force curves shown in Fig. 10 appear different, possibly due to the differences in the tethering position. It might also reflect actual differences in the flies' flight behavior, similar to those found within other data sequences. Because flight just after onset was chosen for the force measurements, the latter appears more likely.

Using static calibration results for dynamic measurements has been a concern in the Drosophila research community. For example, unaccounted for system resonance has unintentionally appeared in flight force data in the past [11]. Although the MEMS microforce sensor behaves as a spring under static loads, it must be modeled as a spring-mass-damper system with dynamic loads applied by the fruit fly. The equation of motion is

$$
\left(m_{1}+m_{2}\right) \ddot{x}+c \dot{x}+k x=F-m_{2} g
$$

where $m_{1}$ is the mass of the movable part of the force sensor, $m_{2}$ is the mass of the fly, $x$ is the displacement, $k$ is the 
sensor stiffness, $F$ is the flight force produced by the fly, and $c=2 \zeta \sqrt{k\left(m_{1}+m_{2}\right)}$ is the damping constant. Dynamic analysis results demonstrate that the spring force component dominates the inertial and damping force components. This ensures strong agreement between flight forces obtained from dynamic analysis and forces obtained from the force sensor using the calibration results under static loads. An average deviation of $0.53 \mu \mathrm{N}$ was calculated. Therefore, the sensor output based on the static calibration results is reliable despite the dynamic loads applied by the fruit fly. The mechanical properties of the sensor also avoid resonance issues or excessive damping. A fast Fourier transform (FFT) analysis on the raw force traces, as shown in Fig. 11, reveals that the signal is composed exclusively of frequencies corresponding to the fundamental (approximately $200 \mathrm{~Hz}$ ) and higher harmonics of the respective wing beat frequencies of the flies. No indication of measurement artifacts due to the dynamic properties of the sensor was observed. The variance between the measured force traces therefore corresponds to differences in the power of the harmonic frequencies, possibly due to differences in the inertial force components elicited by the motion of the wings and/or the thorax.

\section{CONCLUSION}

This paper presents a MEMS microforce sensor with a novel configuration of differential tri-plate comb drives suitable for bulk micromachining that overcomes the difficulty of electrically isolating the two stationary capacitor comb sets in bulk micromachining. A high-yield fabrication process using DRIE on SOI wafers and only three lithographic masks was utilized to construct the high aspect ratio devices. The process features dry release of both suspended structures and the entire device in order to protect fragile components. The MEMS force sensor provides a high sensitivity, broad range, small physical size, robustness, and the suitable geometries. The fruit fly flight force measurement results demonstrate the effectiveness of this technique for reliable and precise real-time measurements of flight forces in tethered flying fruit flies, promising important technological advance for biomechanical studies. Further detailed studies are required to provide reliable measurements of flight forces under highly controlled experimental conditions. Extending this novel sensor design to multiple axes will allow measurements to be made in unprecedented detail to provide further insight into flight biomechanics.

\section{REFERENCES}

[1] G. M. Nelson and R. D. Quinn, "Posture control of a cockroach-like robot," IEEE Contr. Syst. Mag., vol. 19, no. 2, pp. 9-14, 1999.

[2] J. E. Clark, J. G. Cham, S. A. Bailey, E. M. Froehlich, P. K. Nahata, R. J. Full, and M. R. Cutkosky, "Biomimetic design and fabrication of a hexapedal running robot," in The IEEE International Conference on Robotics and Automation, Seoul, Korea, May 21-26, 2001, pp. 3643-3649.

[3] M. C. Birch, R. D. Quinn, G. Hahm, S. M. Phillips, B. T. Drennan, A. J. Fife, R. D. Beer, X. Yu, S. L. Garverick, S. Laksanacharoen, A. J. Pollack, and R. E. Ritzmann, "Cricket-based robots," IEEE Robot. Automat. Mag., vol. 9, no. 4, pp. 20-30, 2002.
[4] M. C. Montesi, B. Martini, A. Pellegrinetti, P. Dario, L. Lencioni, and A. Montano, "An SMA-based flexible active endoscope for minimal invasive surgery," J. Micromech. Microeng., vol. 5, pp. 180-182, 1995.

[5] I. Shimoyama, H. Miura, K. Suzuki, and Y. Ezura, "Insect-like microrobots with external skeletons," IEEE Control Syst. Mag., vol. 13, no. 1, pp. $37-41,1993$.

[6] M. Sitti, "Piezoelectrically actuated four-bar mechanism with two flexible links for micromechanical flying insect thorax," IEEE/ASME Trans. Mechatronics, vol. 8, pp. 26-36, 2003.

[7] M. H. Dickinson and M. S. Tu, "The function of dipteran flight muscle," Comparative Biochem. Phys., vol. 116, no. 3, pp. 223-238, 1997.

[8] S. N. Fry, R. Sayaman, and M. H. Dickinson, "The aerodynamics of free-flight maneuvers in Drosophila," Science, vol. 300, pp. 495-498, 2003.

[9] J. Yan, R. Wood, S. Avandhanula, M. Sitti, and R. S. Fearing, "Toward flapping wing control for a micromechanical flying insect," in Proc. IEEE International Conference on Robotics and Automation, Seoul, Korea, May 21-26, 2001, pp. 3901-3908.

[10] M. H. Dickinson, F. O. Lehmann, and S. P. Sane, "Wing rotation and the aerodynamic basis of insect flight," Science, vol. 284, pp. 1954-1960, 1999.

[11] M. H. Dickinson and K. G. Götz, "The wake dynamics and flight forces of the fruit fly Drosophila melanogaster," J. Exp. Biol., vol. 119, pp. 2085-2104, 1996.

[12] W. C. Tang, T. H. Nguyen, M. W. Judy, and R. T. Howe, "Electrostatic comb drive of lateral polysilicon resonators," Sens. Actuators A, Phys., vol. 21, pp. 328-331, 1990.

[13] Y. Sun, B. J. Nelson, D. P. Potasek, and E. Enikov, "A bulk microfabricated multi-axis capacitive cellular force sensor using transverse comb drives," J. Micromech. Microeng., vol. 12, no. 6, pp. 832-840, 2002.

[14] F. Goodenough, "Airbags boom when IC accelerometer sees $50 \mathrm{~g}$," Electron. Design, vol. 39, pp. 45-56, 1991.

[15] B. P. van Drieënhuizen, N. I. Maluf, I. E. Opris, and G. T. A. Kovacs, "Force-balanced accerometer with $\mathrm{mG}$ resolution, fabricated using silicon fusion bonding and deep reactive ion etching," in Proc. International Conference on Solid-State Sensors and Actuators, Chicago, IL, Jun. 16-19, 1997, pp. 1229-1230.

[16] Y. Xu, S. A. Miller, and N. C. MacDonald, "Microelectromechanical scanning tunneling microscope," in The 8th International Conference on Solid-State Sensors and Actuators, Stockholm, Sweden, Jun. 25-29, 1995, pp. 640-643.

[17] A. Selvakumar and K. Najafi, "A high-sensitivity Z-axis capacitive silicon microaccelerometer with a torsional suspension," J. Microelectromech. Syst., vol. 7, pp. 192-200, 1998.

[18] W. H. Juan and S. W. Pang, "Released Si microstructures fabricated by deep etching and shallow diffusion," J. Microelectromech. Syst., vol. 5, pp. 18-23, 1996.

[19] S. Lee, S. Park, and D. Cho, "The surface/bulk micromachining (SBM) process: A new method for fabricating released MEMS in single crystal silicon,” J. Microelectromech. Syst., vol. 8, pp. 409-416, 1999.

[20] S. Takimoto, R. Kondo, K. Suzuki, and S. Sugiyama, "Fabrication of micromotors using LIGA process," in International Symposium on Micromechatronics and Human Science, Nagoya, Japan, Nov. 24-26, 1999, pp. 221-226.

[21] D. A. Horsley, A. Singh, A. P. Pisano, and R. Horowitz, "Angular micropositioner for disk drives," in Proc. IEEE 10th International Workshop on Micro Electro Mechanical Systems, Nagoya, Japan, Jan. 29-31, 1997, pp. 454-459.

[22] C. H. Hsu and M. A. Schmidt, "Micromachined structures fabricated using a wafer bonded sealed cavity process," in Proc. IEEE Solid-State Sensors Workshop, Hilton Head, SC, 1994, pp. 151-155.

[23] E. H. Klaassen, K. Petersen, J. M. Noworolski, J. Logan, N. I. Maluf, J. Brown, C. Storment, W. Mcculley, and G. T. Kovacs, "Silicon fusion bonding and deep reactive ion etching; A new technology for microstructures," in Proc. The 8th International Conference on Solid-State Sensors and Actuators, Stockholm, Sweden, Jun. 25-29, 1995, pp. 556-559.

[24] J. M. Noworolski, E. Klaassen, J. Logan, K. Petersen, and N. Maluf, "Fabrication of SOI wafers with buried cavities using silicon fusion bonding and electrochemical etchback," in Proc. The 8th International Conference on Solid-State Sensors and Actuators, Stockholm, Sweden, June 25-29, 1995, pp. 71-74.

[25] L. F. Tammero and M. H. Dickinson, "Collision-avoidance and landing responses are mediated by separate pathways in the fruit fly_Drosophila melanogaster," J. Exp. Biol., vol. 205, pp. 2785-2798, 2002. 


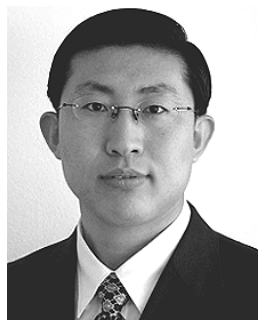

Yu Sun (M'00) received the B.S. degree in electrical engineering from the Dalian University of Technology, China, in 1996, the M.S. degree from the Institute of Automation, Chinese Academy of Sciences, Beijing, China, in 1999, and the Ph.D. degree in mechanical engineering from the University of Minnesota, Minneapolis, in 2003.

He held a Research Scientist position at the Swiss Federal Institute of Technology (ETH-Zurich) before joining the faculty of the Department of Mechanical and Industrial Engineering at the University of Toronto in July, 2004, where he is an Assistant Professor. His research areas are MEMS design, fabrication and testing, control of microstructures, microrobotics, microrobotic biomanipulation, biomechanics, and nanofabrication and nanomanipulation.

Dr. Sun is a Member of the American Society of Mechanical Engineers (ASME).

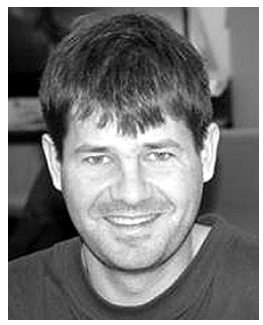

Steven N. Fry received the diploma in 1994 and the Ph.D. degree in natural sciences in 1999 from the University of Zurich, Switzerland.

He continued as a Postdoctoral Associate with D. Robert at the University of Zurich, Switzerland (1999-2000) and M. H. Dickinson at the University of California, Berkeley (2000-2002). He is currently working as a Senior Researcher at the Institute of Neuroinformatics (INI) at the Swiss Federal Institute of Technology (ETH) and University of Zurich, where he leads a work group researching the biomechanics and sensory processing in fruit flies.

D. P. Potasek, photograph and biography not available at the time of publication.

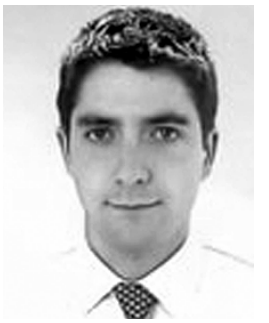

Dominik J. Bell studied Aerospace Engineering at the University of Bristol, U.K., and at Purdue University, West Lafayette, IN, and received the M.Eng. degree from the University of Bristol in 2002. In 2003, he received the M.Phil. degree from the University of Cambridge, U.K., for his research in MEMS actuators and sensors and in micromechanical testing.

In the same year, he joined the Institute of Robotics and Intelligent Systems (IRIS) at the Swiss Federal Institute of Technology (ETH) Zurich, Switzerland, as a Ph.D. student with a research focus on MEMS and NEMS devices and on wireless sensing.

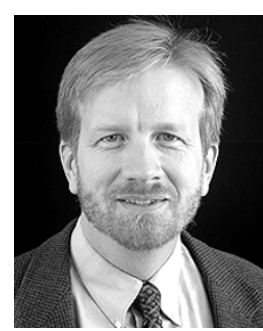

Brad J. Nelson (S'91-M'96) received the B.S. degree in mechanical engineering from the University of Illinois, Chicago, in 1984, the M.S. degree in mechanical engineering University of Minnesota, Minneapolis, in 1987, and the Ph.D. degree in robotics from (School of Computer Science) Carnegie Mellon University, Pittsburgh, PA, in 1995.

$\mathrm{He}$ is the Professor of Robotics and Intelligent Systems at the Swiss Federal Institute of Technology (ETH), Zurich, and heads the Institute of Robotics and Intelligent Systems there. He has been on the faculty of the University of Minnesota and the University of Illinois at Chicago, has worked at Motorola and Honeywell, and has served as a United States Peace Corps Volunteer in Botswana, Africa. His most recent scientific contributions have been in the area of microrobotics and biomicrorobotics, including efforts in robotic micromanipulation, microassembly, MEMS (sensors and actuators), mechanical manipulation of biological cells and tissue, and nanofabrication. He has also contributed to the fields of visual servoing, force control, sensor integration, and web-based control and programming of robots.

Dr. Nelson is a Member of the American Society of Mechanical Engineers (ASME). He has been awarded a McKnight Land-Grant Professorship and is a recipient of the Office of Naval Research Young Investigator Award, the National Science Foundation Faculty Early Career Development (CAREER) Award, the McKnight Presidential Fellows Award, and the Bronze Tablet. He was elected as a Robotics and Automation Society Distinguished Lecturer $\langle$ http://www.ncsu.edu/IEEE-RAS/RAS/DistLect.html〉 in 2003 and received the Best Conference Paper Award at the IEEE 2004 International Conference on Robotics and Automation. Professor Nelson serves on or has been a member of the editorial boards of the IEEE TRANSACTIONS ON ROBOTICS, the Journal of Micromechatronics and the IEEE RoBotics AND AUTOMATION MAGAZINE. He has chaired several international workshops and conferences. 